\title{
Belarus Peatland Restoration Project Database
}

\author{
Annett Thiele, Nadzeya Liashchynskaya, Tatsiana Broska, Susanne Bärisch, Arkadi \\ Skuratovich, Dimitri Dubovik, Jazep Stepanovich, Galina Ermolenko, Oleg Sozinov \& \\ Anastasia Sakovich
}

\begin{abstract}
The project 'Restoring Peatlands and applying Concepts for Sustainable Management in Belarus' supported by the International Climate Initiative of the German Federal Ministry for the Environment, Nature Conservation and Nuclear Safety implements a large scale peatland investigation and rewetting programme in Belarus (2008-2012). Huge degraded peatlands (ca 15,000 ha) are monitored before and after rewetting. To be able to account for the emission reductions upon rewetting, a search for a proxy for greenhouse gas (GHG) emissions revealed that water level fluctuations are the main influencing factor for emissions. The indication function of species and species groups can provide information about mean water levels on peatlands. Therefore vegetation types are worked out and tested as a proxy to estimate GHG emissions. The investigations include full relevés, long term water level fluctuations and partly GHG measurements using the chamber method on a range from natural, semi-natural and degraded fens and bogs. A model (GEST-Model) based on space for time substitution will allow to monitor GHG emissions from peatlands before and after rewetting and to account for the emissions saved under rewetting and restoring peatlands. This report describes the available content in the Belarus Peatland Restoration Project Database (GIVD ID EU-BY-001).
\end{abstract}

Keywords: bog; fen; restoration; rewetting; vegetation indication.

\section{Belarus Peatland Restoration Project Database}

Scope: Full vegetation releves on natural, drained and restored peatlands (fen and bog sites) to test and verify the GEST (Greenhouse Gas Emission Site Types)-model (Couwenberg et al. 2011) for Belarus.

Status: completed and continuing

Period: 2009-2012

Database manager(s): Annett Thiele (annetthiele@gmail.com); Nadzeya Liashchynskaya (nadzeya.liashchynskaya@gmail.com); Tatsiana Broska (bronibroska@mail.ru); Anastasia Sakovich (anastasia_pryaz@inbox.ru)

Owner: APB BirdLife partner Belarus

Web address: http://www.restoringpeatlands.org, http://www.ptushki.org

Availability: free upon request

Online upload: [NA]

Database format(s): MS Access

Export format(s): [NA]

Publication: [NA]

Plot type(s): normal plots

Non-overlapping plots: 900

Plot-size range: $0.64-25 \mathrm{~m}^{2}$

Total plot observations: 900

Estimate of existing plots: 450

Number of sources: [NA]
Online search: [NA]

Completeness: $200 \%$

Valid taxa: [NA]

Countries: BY: $100.0 \%$

Forest: $0 \%$ - Non-forest: aquatic: $0 \%$; semi-aquatic: $50 \%$; arctic-alpine: $0 \%$; natural: $0 \%$; semi-natural: $0 \%$; anthropogenic: $50 \%$

Guilds: all vascular plants: $100 \%$; bryophytes (terricolous or aquatic): $100 \%$

Environmental data: soil $\mathrm{pH}$ : $80 \%$; other soil attributes: $30 \%$; land use categories: $30 \%$

Performance measure(s): cover: $100 \%$; measurements like diameter or height of trees: $100 \%$

Geographic localisation: GPS coordinates (precision $25 \mathrm{~m}$ or less): $100 \%$

Sampling periods: [NA]

Information as of 2012-07-12; further details and future updates available from http://www.givd.info/ID/EU-BY-001

Annett Thiele* (annettthiele@gmail.com), Nadzeya Liashchynskaya (nadzeya.liashchynskaya@gmail.com)

Department of Sustainable Peatland Management, APB BirdLife Partner Belarus, Makaenka 8-313, 220014 Minsk, BELARUS

Tatsiana Broska (bronibroska@mail.ru), Arkadi Skuratovich (flora@biobel.bas-net.by), Dimitri Dubovik (flora@biobel.bas-net.by),

Jazep Stepanovich (jazep@ biobel.bas-net.by), Galina Ermolenko (flora@biobel.bas-net.by)

Institute of Experimental Botany, National Academy of Sciences, Akademichnaya 27, 220072 Minsk, BELARUS

Susanne Bärisch (susanne.baerisch@succow-stiftung.de)

Michael Succow Foundation, Ellernholzstr. 1/3, 17489 Greifswald, GERMANY 
Oleg Sozinov (o.sozinov@grsu.by), Anastasia Sakovich (anastasia_pryaz@inbox.ru)

Institute of Botany, State University Grodno, Dovatora 3/1-139, 143, 230000 Grodno, BELARUS

*Corresponding author 